\title{
MIXED QUASI INVEX EQUILIBRIUM PROBLEMS
}

\author{
MUHAMMAD ASLAM NOOR
}

Received 11 October 2003

\begin{abstract}
We introduce a new class of equilibrium problems, known as mixed quasi invex equilibrium (or equilibrium-like) problems. This class of invex equilibrium problems includes equilibrium problems, variational inequalities, and variational-like inequalities as special cases. Several iterative schemes for solving invex equilibrium problems are suggested and analyzed using the auxiliary principle technique. It is shown that the convergence of these iterative schemes requires either pseudomonotonicity or partially relaxed strong monotonicity, which are weaker conditions than the previous ones. As special cases, we also obtained the correct forms of the algorithms for solving variational-like inequalities, which have been considered in the setting of convexity. In fact, our results represent significant and important refinements of the previously known results.
\end{abstract}

2000 Mathematics Subject Classification: 49J40, 90C26.

1. Introduction. It is well known that the equilibrium problems theory provides us with a unified, natural, innovative, and general framework to study various unrelated problems arising in finance, economics, network analysis, transportation, elasticity, and nonlinear optimization; see [3, 10, 11, 12, 19, 21, 27, 29, 34, 22, 36] and the references therein. It has been shown that equilibrium problems include variational inequalities and optimization problems as special cases. It is worth mentioning that almost all the results obtained so far are in the setting of convexity. It has been noted that these results may not hold in the invex setting. Inspired and motivated by the research going on in this interesting and fascinating area, we introduce and investigate a new class of equilibrium problems, which is called invex equilibrium (or equilibrium-like) problems in the setting of invexity. It has been shown that invex equilibrium problems include variational-like inequalities, equilibrium problems, and variational inequalities as special cases. Hence, collectively, the invex equilibrium problems cover a vast range of applications. A significant generalization of convex functions is that of invex functions introduced by Hanson [14]. Hanson's initial result inspired a great deal of subsequent work which has greatly expanded the role and applications of invexity in nonlinear optimization and other branches of pure and applied sciences. Weir and Mond [38] and Noor [23, 24, 25] have studied the basic properties of the preinvex functions and their role in optimization and variational-like inequalities. It is well known that the preinvex functions and invex sets may not be convex functions and convex sets. Noor [23] has proved that the minimum of the differentiable preinvex (invex) functions on the invex sets in normed spaces can be characterized by a class of variational inequalities, known as variational-like (prevariational) inequalities. Thus it is clear that the concept 
of invexity plays exactly the same role in variational-like inequalities that classical convexity plays in variational inequalities. This shows that the variational-like inequalities are well defined in the setting of invexity. Ironically, we note that all the results for variational-like inequalities are being obtained in the setting of classical convexity; see $[1,6,7,9,15,18,37]$ and the references therein. No attempt has been made to utilize the concept of invexity. Since the preinvex and invex functions are not convex functions, all these results for variational-like inequalities are misleading, since these results have been obtained using the KKM mappings and diagonal convexity. It is still an open problem to prove that the subdifferential of a differentiable preinvex function is a maximal monotone operator. This implies that one cannot define the resolvent operator associated with the proper, preinvex, and lower-semicontinuous functions as it has been defined in $[6,18]$. In brief, we would like to emphasize the fact that variational-like inequalities must be studied in the setting of invexity. There is very delicate and subtle difference between the concepts of invexity and convexity, which should be taken into account while considering variational-like inequalities and related optimization problems.

There are a substantial number of numerical methods including projection technique and its variant forms, Wiener-Hopf equations, and auxiliary principle and resolvent equations methods for solving variational inequalities. However, it is known $[22,24,25,27,28,29,34,35]$ that projection technique, Wiener-Hopf equations, and proximal and resolvent equations techniques cannot be extended and generalized to suggest and analyze similar iterative methods for solving invex equilibrium problems and variational-like inequalities due to the presence of the function $\eta(\cdot, \cdot)$. This fact motivated the use the auxiliary principle technique, which is mainly due to Glowinski et al. [13], to suggest and analyze some iterative schemes for solving invex equilibrium problems. The main and basic idea in this technique is to consider an auxiliary invex equilibrium problem related to the original problem. This way one defines a mapping connecting the solutions of both these problems. In this case, one has to show that the mapping connecting the solution is a contraction mapping and consequently it has a fixed point, which is the solution of the original problem. This technique has been used to suggest and analyze a number of iterative methods for solving various classes of equilibrium problems, variational-like inequalities, and variational inequalities. It has been shown that a substantial number of numerical methods can be obtained as special cases from this technique; see [22, 24, 25, 27, 28, 29, 34, 35]. We prove that the convergence of these methods requires either pseudomonotonicity or partially relaxed strong monotonicity. In this respect, our results represent an improvement of the previously known results. Our results can be considered as a novel and important application of the auxiliary principle technique. Since the invex equilibrium problems with bifunction include several classes of variational-like inequalities, variational inequalities, and equilibrium and related optimization problems as special cases, results obtained in this paper continue to hold for these problems.

2. Preliminaries. Let $H$ be a real Hilbert space, whose inner product and norm are denoted by $\langle\cdot, \cdot\rangle$ and $\|\cdot\|$, respectively. Let $K$ be a nonempty closed set in $H$. Let $f: K \rightarrow$ $H$ and $\eta(\cdot, \cdot): K \times K \rightarrow H$ be continuous functions. 
First of all, we recall the following well-known results and concepts.

Definition 2.1 [38]. Let $u \in K$. Then the set $K$ is said to be invex at $u$ with respect to $\eta(\cdot, \cdot)$ if, for all $u, v \in K, t \in[0,1]$,

$$
u+\operatorname{t\eta }(v, u) \in K
$$

$K$ is said to be an invex set with respect to $\eta$ if $K$ is invex at each $u \in K$. The invex set $K$ is also called an $\eta$-connected set.

From now onward $K$ is a nonempty closed invex set in $H$ with respect to $\eta(\cdot, \cdot)$, unless otherwise specified.

Definition 2.2 [38]. The function $f: K \rightarrow H$ is said to be preinvex with respect to $\eta$ if, for all $u, v \in K, t \in[0,1]$,

$$
f(u+\operatorname{t\eta }(v, u)) \leq(1-t) f(u)+t f(v) .
$$

The function $f: K \rightarrow H$ is said to be preconcave if and only if $-f$ is preinvex.

DeFINITION 2.3. The differentiable function $f: K \rightarrow H$ is said to be an invex function with respect to $\eta(\cdot, \cdot)$ if, for all $u, v \in K$,

$$
f(v)-f(u) \geq\left\langle f^{\prime}(u), \eta(v, u)\right\rangle,
$$

where $f^{\prime}(u)$ is the differential of $f$ at $u$. Note that Hanson [14] defined the concept of invex differentiable functions on the whole space. The concepts of the invex and preinvex functions have played a very important role in the development of convex programming. From Definitions 2.2 and 2.3, it is clear that the differentiable preinvex functions are invex functions, but the converse is not true [38]. However, Mohan and Neogy [20] have shown that a differentiable function which is invex on an invex set $K$ is also a preinvex function under some conditions.

Definition 2.4. A function $f$ is said to be a strongly preinvex function on $K$ with respect to the function $\eta(\cdot, \cdot)$ with modulus $\mu$ if, for all $u, v \in K, t \in[0,1]$,

$$
f(u+t \eta(v, u)) \leq(1-t) f(u)+t f(v)-t(1-t) \mu\|\eta(v, u)\|^{2} .
$$

Clearly, the differentiable strongly preinvex function $F$ is a strongly invex function with module constant $\mu$, that is,

$$
f(v)-f(u) \geq\left\langle f^{\prime}(u), \eta(v, u)\right\rangle+\mu\|\eta(v, u)\|^{2},
$$

but the converse is not true in general. 
DEFINITION 2.5. The bifunction $\varphi(\cdot, \cdot): H \times H \rightarrow R \cup\{+\infty\}$ is called skew-symmetric if and only if

$$
\varphi(u, u)-\varphi(u, v)-\varphi(v, u)-\varphi(v, v) \geq 0, \quad \forall u, v \in H
$$

Clearly if the skew-symmetric bifunction $\varphi(\cdot, \cdot)$ is bilinear, then $\varphi(u, u) \geq 0, \forall u \in H$. In fact,

$$
\varphi(u, u)-\varphi(u, v)-\varphi(v, u)+\varphi(v, v)=\varphi(u-v, u-v) \geq 0, \quad \forall u, v \in H
$$

For a given continuous function $F(\cdot, \cdot): K \times K \rightarrow R$ and continuous bifunction $\varphi(\cdot, \cdot)$ : $K \times K \rightarrow R \cup\{\infty\}$, consider the problem of finding $u \in K$ such that

$$
F(u, v)+\varphi(v, u)-\varphi(u, u) \geq 0, \quad \forall v \in K
$$

which is called the mixed quasi invex equilibrium problem, where the set $K$ is an invex set in $H$.

If $F(u, v) \equiv\langle T u, \eta(v, u)\rangle$, where $T: H \rightarrow H$, and $\eta(\cdot, \cdot): K \times K \rightarrow R \cup\{\infty\}$, then problem (2.8) is equivalent to finding $u \in K$ such that

$$
\langle T u, \eta(v, u)\rangle+\varphi(v, u)-\varphi(u, u) \geq 0, \quad \forall v \in K,
$$

which is known as the mixed quasi-variational-like inequality problem. Problem (2.9) and its variant forms have been studied extensively by many authors in the setting of convexity using the KKM mappings and fixed-point theory; see [1, 6, 7, 9, 15, 18, 37]. It is worth mentioning that the concept of variational-like inequalities in the convexity setting is not well defined and consequently all the results so far obtained in the convexity (scalar and vector) are misleading.

If $\eta(v, u)=v-u$, then the invex set $K$ becomes the convex set and problem (2.8) is called the mixed quasi-equilibrium problem of finding $u \in K$ such that (2.8) holds which was introduced and studied by Noor [29].

Also the variational-like inequality (2.9) is equivalent to finding $u \in K$ such that

$$
\langle T u, v-u\rangle+\varphi(v, u)-\varphi(u, u) \geq 0, \quad \forall v \in K,
$$

which is known as the mixed quasivariational inequality.

In brief, for suitable and appropriate choice of the operators and spaces, one can obtain a number of new and known classes of equilibrium, variational-like inequalities, and variational inequalities as special cases of problem (2.8). This clearly shows that problem (2.8) is a quite general and unifying one. For the recent applications, numerical methods, and formulations of variational inequalities, variational-like inequalities, and equilibrium problems; see $[1,2,3,4,6,5,7,8,9,10,11,12,13,14,15,16,17,18,19$, 20, 21, 22, 23, 24, 25, 26, 27, 28, 29, 30, 31, 32, 33, 34, 35, 36, 37, 38, 39, 40]. 
DEFINITION 2.6. The function $F(\cdot, \cdot): K \times K \rightarrow R$ is said to be

(i) pseudomonotone with respect to the bifunction $\varphi(\cdot, \cdot)$, if

$$
\begin{aligned}
& F(u, v)+\varphi(v, u)-\varphi(u, u) \geq 0 \\
& \quad \Longrightarrow-F(v, u)+\varphi(v, u)-\varphi(u, u) \geq 0, \quad \forall u, v \in K
\end{aligned}
$$

(ii) partially relaxed strong monotone, if there exists a constant $\alpha>0$ such that

$$
F(u, v)+F(v, z) \leq \alpha\|\eta(z, u)\|^{2}, \quad \forall u, v, z \in K
$$

(iii) hemicontinuous, for all $u, v \in K$ and $t \in[0,1]$, if the mapping $F(u+t \eta(v, u), v)$ is continuous.

Note that for $z=u$, partially relaxed strong monotonicity reduces to

$$
F(u, v)+F(v, u) \leq 0, \quad \forall u, v \in K,
$$

which is known as the monotonicity of $F(\cdot, \cdot, \cdot)$.

For $F(u, v)=\langle T u, \eta(v, u)\rangle$ and $F(u, v)=\langle T u, v-u\rangle$, Definition 2.6 reduces to the well-known concepts in variational inequalities theory, see [28].

LEMMA 2.7. Let the function $F(\cdot, \cdot)$ be pseudomonotone and hemicontinuous. If the function $F(\cdot, \cdot)$ is preinvex with respect to the second argument and the function $\varphi(\cdot, \cdot)$ is preinvex with respect to the first argument, then problem (2.8) is equivalent to finding $u \in K$ such that

$$
F(v, u)+\varphi(u, u)-\varphi(v, u) \leq 0, \quad \forall v \in K
$$

Proof. Let $u \in K$ be a solution of invex equilibrium problem (2.8). Then

$$
F(u, v)+\varphi(v, u)-\varphi(u, u) \geq 0, \quad \forall v \in K
$$

implies

$$
F(v, u)+\varphi(u, u)-\varphi(v, u) \leq 0, \quad \forall v \in K
$$

since the function $F(\cdot, \cdot)$ is pseudomonotone with respect to the function $\varphi(\cdot, \cdot)$.

Since $K$ is an invex set, for all $u, v \in K, t \in[0,1]$, there exists an operator $\eta(\cdot, \cdot)$ such that $v_{t}=u+t \eta(v, u) \in K$. Taking $v=v_{t}$ in (2.16) we have

$$
F\left(v_{t}, u\right)+\varphi(u, u)-\varphi\left(v_{t}, u\right) \leq 0, \quad \forall v_{t} \in K
$$

from which we have

$$
F\left(v_{t}, u\right) \leq t\{\varphi(v, u)-\varphi(u, u)\}
$$


using the preinvexity of $\varphi(\cdot, \cdot)$ in the first argument. Now

$$
\begin{aligned}
0 & \leq F\left(v_{t}, v_{t}\right) \leq t F\left(v_{t}, v\right)+(1-t) F\left(v_{t}, u\right) \\
& \leq t F\left(v_{t}, v\right)+t(1-t)\{\varphi(v, u)-\varphi(u, u)\} \quad(\operatorname{using}(2.18)) .
\end{aligned}
$$

Dividing inequality (2.19) by $t$ and taking the limit as $t \rightarrow 0$, since $F(\cdot, \cdot)$ is hemicontinuous, we have (2.15) which shows that $u \in K$ is a solution of (2.8), the required result.

REMARK 2.8. Problem (2.14) is called dual mixed quasi invex equilibrium problem. One can easily show that the solution set of (2.14) is an invex and closed set in $H$. Lemma 2.7 can be viewed as a natural extension and generalization of Minty's lemma; see $[2,28]$.

3. Main results. In this section, we use the auxiliary principle technique to suggest and analyze some iterative algorithms for solving invex equilibrium problem (2.8). For a given $u \in K$, consider the problem of finding a unique $w \in K$ such that

$$
\rho F(w, v)+\left\langle E^{\prime}(w)-E^{\prime}(u), \eta(v, w)\right\rangle \geq \rho\{\varphi(w, w)-\varphi(v, w)\} \quad \forall v \in K,
$$

which is known as the auxiliary invex equilibrium problem. Here $E^{\prime}(u)$ is the differential of a strong preinvex function $E(u)$ at the point $u \in K$. Problem (3.1) has a unique solution, since the function $E$ is a strongly preinvex function. We remark that if $w=u$, then $w$ is a solution of (2.8). On the basis of this observation, we suggest and analyze the following iterative algorithm for solving (2.8) as long as (3.1) is easier to solve than (2.8).

Algorithm 3.1. For a given $u_{0} \in H$, calculate the approximate solution $u_{n+1}$ by the iterative scheme

$$
\begin{aligned}
\rho F\left(u_{n+1}, v\right)+ & \left\langle E^{\prime}\left(u_{n+1}\right)-E^{\prime}\left(u_{n}\right), \eta\left(v, u_{n+1}\right)\right\rangle \\
& \rho\left\{\varphi\left(v, u_{n+1}\right)-\varphi\left(u_{n+1}, u_{n+1}\right)\right\} \geq 0, \quad \forall v \in K .
\end{aligned}
$$

Algorithm 3.1 is called the proximal point method for solving mixed quasi invex equilibrium problem (2.8). Note that if $\eta(v, u)=v-u$, then Algorithm 3.1 reduces to the following method for solving mixed quasi-equilibrium problem (2.8), which is due to Noor [28].

Algorithm 3.2. For a given $u_{0} \in H$, find the approximate solution $u_{n+1}$ by the iterative scheme

$$
\begin{aligned}
\rho F\left(u_{n+1}, v\right) & +\left\langle E^{\prime}\left(u_{n+1}\right)-E^{\prime}\left(u_{n}\right), v-u_{n+1}\right\rangle \\
& +\rho\left\{\varphi\left(v, u_{n+1}\right)-\varphi\left(u_{n+1}, u_{n+1}\right)\right\} \geq 0, \quad \forall v \in K,
\end{aligned}
$$

which is called the proximal method. Note that $E^{\prime}(u)$ is the differential of a strongly convex function $E(u)$ at $u \in K$, a convex set in $H$.

If $F(u, v)=\langle T u, \eta(v, u)\rangle$, then Algorithm 3.1 collapses to the following method for solving mixed quasi-variational-like inequalities (2.9). 
AlgORITHm 3.3. For a given $u_{0} \in H$, calculate the approximate solution $u_{n+1}$ by the iterative scheme

$$
\begin{aligned}
& \left\langle\rho T u_{n+1}+E^{\prime}\left(u_{n+1}\right)-E^{\prime}\left(u_{n}\right), \eta\left(v, u_{n+1}\right)\right\rangle \\
& \quad \times \rho\left\{\varphi\left(v, u_{n+1}\right)-\varphi\left(u_{n+1}, u_{n+1}\right)\right\} \geq 0 \quad \forall v \in K .
\end{aligned}
$$

Here $E^{\prime}(u)$ is the differential of a differentiable strongly preinvex function $E(u)$ at a point $u \in K$, an invex set in $H$. Algorithm 3.3 can be considered as a correct algorithm for solving variational-like inequalities (2.9). Note that all the algorithms and their analyses in $[1,4,6,5,7,9,15,18,37]$ are proposed and investigated in the setting of convexity. Consequently their algorithms and results are wrong. As we have pointed out earlier, the variational-like inequalities are only well defined in the setting of invexity. In view of these facts and comments, results obtained [1, 4, 6, 5, 7, 9, 15, 18, 37] must be modified and studied in the setting of invexity. In a similar way, one can obtain the proximal point method for solving classical equilibrium problems, variational inequalities, and related optimization problems.

We now study the convergence criteria of Algorithm 3.1. The analysis is in the spirit of [29, 31]. We need the following condition.

Assumption 3.4. For all $u, v, z \in H$, the function $\eta(\cdot, \cdot)$ satisfies the condition

$$
\eta(u, v)=\eta(u, z)+\eta(z, v)
$$

Assumption 3.4 has been used to study the existence of a solution of variational-like inequalities by many authors; see $[6,15,18]$. Note that $\eta(u, v)=0$ if and only if $=v$, for all $u, v \in H$.

THEOREM 3.5. Let the function $F(\cdot, \cdot)$ be pseudomonotone. If $E$ is a differentiable strongly preinvex function with modulus $\beta>0$ and (3.5) holds, then the approximate solution $u_{n+1}$ obtained from Algorithm 3.1 converges to a solution $u \in K$ satisfying (2.8).

Proof. Let $u \in K$ be a solution of (2.8). Then

$$
-F(v, u)+\varphi(v, u)-\varphi(u, u) \geq 0, \quad \forall v \in K,
$$

since the bifunction $F(\cdot, \cdot)$ is pseudomonotone with respect to the bifunction $\varphi(\cdot, \cdot)$.

Taking $v=u_{n+1}$ in (3.6), we have

$$
-F\left(u_{n+1}, u\right)+\varphi\left(u_{n+1}, u\right)-\varphi(u, u) \geq 0 .
$$

Consider the function

$$
\begin{aligned}
B(u, z) & =E(u)-E(z)-\left\langle E^{\prime}(z), \eta(u, z)\right\rangle \\
& \geq \beta\|\eta(u, z)\|^{2}, \quad \text { using the strong invexity of } E .
\end{aligned}
$$


Combining (3.5), (3.6), (3.7), and (3.8), we have

$$
\begin{aligned}
B\left(u, u_{n}\right)-B\left(u, u_{n+1}\right)= & E\left(u_{n+1}\right)-E\left(u_{n}\right)-\left\langle E^{\prime}\left(u_{n}\right), \eta\left(u, u_{n}\right)\right\rangle \\
& +\left\langle E^{\prime}\left(u_{n+1}\right), \eta\left(u, u_{n+1}\right)\right\rangle \\
= & E\left(u_{n+1}\right)-E\left(u_{n}\right)-\left\langle E^{\prime}\left(u_{n}\right)-E^{\prime}\left(u_{n+1}\right), \eta\left(u, u_{n+1}\right)\right\rangle \\
& -\left\langle E^{\prime}\left(u_{n}\right), \eta\left(u_{n+1}, u_{n}\right)\right\rangle \\
\geq & \beta\left\|\eta\left(u_{n+1}, u_{n}\right)\right\|^{2}+\left\langle E^{\prime}\left(u_{n+1}\right)-E^{\prime}\left(u_{n}\right), \eta\left(u, u_{n+1}\right)\right\rangle \\
\geq & \beta\left\|\eta\left(u_{n+1}, u_{n}\right)\right\|^{2}-F\left(u_{n+}, u\right) \\
& +\rho\left\{\varphi\left(u_{n+1}, u_{n+1}\right)-\varphi\left(u, u_{n+1}\right)\right\}, \\
\geq & \beta\left\|\eta\left(u_{n+1}, u_{n}\right)\right\|^{2}+\rho\left\{\varphi\left(u_{n+1}, u_{n+1}\right)-\rho \varphi\left(u, u_{n+1}\right)\right. \\
\geq & \left.\beta\left\|\eta\left(u_{n+1}, u_{n}\right)\right\|^{2}, \quad-\varphi\left(u_{n+1}, u\right)+\varphi(u, u)\right\}
\end{aligned}
$$

since the bifunction $\varphi(\cdot, \cdot)$ is skew-symmetric.

If $u_{n+1}=u_{n}$, then clearly $u_{n}$ is a solution of (2.8). Otherwise, the sequence $B\left(u, u_{n}\right)-$ $B\left(u, u_{n+1}\right)$ is nonnegative and we must have

$$
\lim _{n \rightarrow \infty}\left(\left\|\eta\left(u_{n+1}, u_{n}\right)\right\|\right)=0 .
$$

Now essentially using the technique of Zhu and Marcotte [40], it can be shown that the entire sequence $\left\{u_{n}\right\}$ converges to the cluster point $\bar{u}$ satisfying the invex equilibrium problem (2.8).

It is well known that to implement the proximal methods, one has to calculate the approximate solution implicitly, which is in itself a difficult problem. To overcome this drawback, we suggest another iterative method by using the auxiliary principle technique for solving the invex equilibrium problem (2.8).

For a given $u \in K$, consider the problem of a unique $w \in K$ such that

$$
\rho F(u, v)+\left\langle E^{\prime}(w)-E^{\prime}(u), \eta(v, w)\right\rangle \geq \rho\{\varphi(w, w)-\varphi(v, w)\}, \quad \forall v \in K,
$$

which is called the auxiliary invex equilibrium problem. From the strong preinvexity of the differentiable function, it follows that problem (3.11) has a unique solution. Note that problems (3.11) and (3.1) are quite different. It is clear that if $w=u$, then $w$ is a solution of invex equilibrium problem (2.8). This observation enables to suggest and analyze the following iterative method for solving (2.8).

Algorithm 3.6. For a given $u_{0} \in H$, calculate the approximate solution $u_{n+1}$ by the iterative scheme

$$
\begin{aligned}
\rho F\left(u_{n}, v\right) & +\left\langle E^{\prime}\left(u_{n+1}\right)-E^{\prime}\left(u_{n}\right), \eta\left(v, u_{n+1}\right)\right\rangle \\
& +\rho\left\{\varphi\left(v, u_{n+1}\right)-\varphi\left(u_{n+1}, u_{n+1}\right)\right\} \geq 0, \quad \forall v \in K .
\end{aligned}
$$

Note that if $F(u, T u, v)=\langle T u, \eta(v, u)\rangle$, then Algorithm 3.6 reduces to the following iterative method for solving variational-like inequalities (2.9). 
Algorithm 3.7. For a given $u_{0} \in H$, find the approximate solution $u_{n+1}$ by the iterative scheme

$$
\begin{aligned}
& \left\langle\rho T u_{n}+E^{\prime}\left(u_{n+1}\right)-E^{\prime}\left(u_{n}\right), \eta\left(v, u_{n+1}\right)\right\rangle \\
& \quad+\rho\left\{\varphi\left(v, u_{n+1}\right)-\varphi\left(u_{n+1}, u_{n+1}\right)\right\} \geq 0, \quad \forall v \in K .
\end{aligned}
$$

For $\eta(v, u)=v-u$, the invex set $K$ becomes the convex set $K$, and consequently Algorithms 3.6 and 3.7 are exactly the same iterative methods for solving convex equilibrium problems and variational inequalities as that of [28, 29].

One can study the convergence analysis of Algorithm 3.6 using essentially the technique of Theorem 3.5. However, we give its proof for the sake of completeness and to convey an idea.

THEOREM 3.8. Let the function $F(\cdot, \cdot, \cdot)$ be partially relaxed strong monotone with constant $\alpha>0$ and let $E(u)$ be a differentiable strongly preinvex function with modulus $\beta>0$. Let the bifunction $\varphi(\cdot, \cdot)$ be skew-symmetric and let (3.5) hold. If $0<\rho<(\beta / \alpha)$, then the approximate solution $u_{n+1}$ obtained from Algorithm 3.6 converges to a solution $u \in K$ of the invex equilibrium problem (2.8).

Proof. Let $u \in K$ be a solution of (2.8). Then taking $v=u_{n+1}$ in (2.8) and $v=u$ in (3.12), we have

$$
\begin{gathered}
F\left(u, u_{n+1}\right)+\varphi\left(u_{n+1}, u\right)-\varphi(u, u) \geq 0, \\
\rho F\left(u_{n}, u\right)+\left\langle E^{\prime}\left(u_{n+1}\right)-E^{\prime}\left(u_{n}\right), \eta\left(u, u_{n+1}\right)\right\rangle \\
+\rho\left\{\varphi\left(v, u_{n+1}\right)-\varphi\left(u_{n+1}, u_{n+1}\right)\right\} \geq 0 .
\end{gathered}
$$

From (3.5), (3.12), (3.14), and (3.15), we have

$$
\begin{aligned}
B\left(u, u_{n}\right)-B\left(u, u_{n+1}\right) \geq & \beta\left\|\eta\left(u_{n+1}, u_{n}\right)\right\|^{2}+\left\langle E^{\prime}\left(u_{n+1}\right)-E^{\prime}\left(u_{n}\right), \eta\left(u, u_{n+1}\right)\right\rangle \\
\geq & \beta\left\|\eta\left(u_{n+1}, u_{n}\right)\right\|^{2}-\rho\left\{F\left(u, u_{n}\right)+F\left(u_{n+1}, u\right)\right\} \\
& +\rho\left\{\varphi\left(u_{n+1}, u_{n+1}\right)-\varphi\left(u, u_{n+1}\right)\right. \\
& \left.-\varphi\left(u_{n+1}, u\right)+\varphi(u, u)\right\} \\
\geq & \{\beta-\alpha \rho\}\left\|\eta\left(u_{n+1}, u_{n}\right)\right\|^{2},
\end{aligned}
$$

where we have used the fact that the function $F(\cdot, \cdot)$ is partially relaxed strong monotone with constant $\alpha>0$ and the bifunction $\varphi(\cdot, \cdot)$ is skew-symmetric.

If $u_{n+1}=u_{n}$, then clearly $u_{n}$ is a solution of (2.8). Otherwise, for $0<\rho<(\beta / \alpha)$, the sequence $B\left(u, u_{n}\right)-B\left(u, u_{n+1}\right)$ is nonnegative and we must have

$$
\lim _{n \rightarrow \infty}\left(\left\|\eta\left(u_{n+1}, u_{n}\right)\right\|\right)=0
$$

Now by using the technique of Zhu and Marcotte [40], it can be shown that the entire sequence $\left\{u_{n}\right\}$ converges to the cluster point $\bar{u}$ satisfying the mixed quasi invex equilibrium problem (2.8). 


\section{REFERENCES}

[1] Q. H. Ansari and J.-C. Yao, Iterative schemes for solving mixed variational-like inequalities, J. Optim. Theory Appl. 108 (2001), no. 3, 527-541.

[2] C. Baiocchi and A. Capelo, Variational and Quasivariational Inequalities, John Wiley \& Sons, New York, 1984.

[3] E. Blum and W. Oettli, From optimization and variational inequalities to equilibrium problems, Math. Student 63 (1994), no. 1-4, 123-145.

[4] O. Chadli, X. Q. Yang, and J.-C. Yao, On generalized vector pre-variational and prequasivariational inequalities, J. Math. Anal. Appl. 295 (2004), no. 2, 392-403.

[5] X. P. Ding, Iterative algorithms of solutions for generalized mixed implicit equilibrium-like problems, to appear in Appl. Math. Comput.

[6] __ Generalized quasi-variational-like inclusions with nonconvex functionals, Appl. Math. Comput. 122 (2001), no. 3, 267-282.

[7] X. P. Ding and C. L. Luo, Perturbed proximal point algorithms for general quasi-variationallike inclusions, J. Comput. Appl. Math. 113 (2000), no. 1-2, 153-165.

[8] N. El Farouq, Pseudomonotone variational inequalities: convergence of proximal methods, J. Optim. Theory Appl. 109 (2001), no. 2, 311-326.

[9] Y. P. Fang and N.-J. Huang, Variational-like inequalities with generalized monotone mappings in Banach spaces, J. Optim. Theory Appl. 118 (2003), no. 2, 327-338.

[10] F. Flores-Bazán, Existence theorems for generalized noncoercive equilibrium problems: the quasi-convex case, SIAM J. Optim. 11 (2000), no. 3, 675-690.

[11] F. Giannessi and A. Maugeri (eds.), Variational Inequalities and Network Equilibrium Problems, Plenum Press, New York, 1995.

[12] F. Giannessi, A. Maugeri, and P. M. Pardalos (eds.), Equilibrium Problems: Nonsmooth Optimization and Variational Inequality Methods, Nonconvex Optimization and its Applications, vol. 58, Kluwer Academic Publishers, Dordrecht, 2001.

[13] R. Glowinski, J.-L. Lions, and R. Trémolières, Numerical Analysis of Variational Inequalities, Studies in Mathematics and its Applications, vol. 8, North-Holland Publishing, Amsterdam, 1981.

[14] M. A. Hanson, On sufficiency of the Kuhn-Tucker conditions, J. Math. Anal. Appl. 80 (1981), no. 2, 545-550.

[15] N.-J. Huang and C.-X. Deng, Auxiliary principle and iterative algorithms for generalized set-valued strongly nonlinear mixed variational-like inequalities, J. Math. Anal. Appl. 256 (2001), no. 2, 345-359.

[16] N. Kikuchi and J. T. Oden, Contact Problems in Elasticity: A Study of Variational Inequalities and Finite Element Methods, SIAM Studies in Applied Mathematics, vol. 8, SIAM, Philadelphia, 1988.

[17] D. Kinderlehrer and G. Stampacchia, An Introduction to Variational Inequalities and Their Applications, Classics in Applied Mathematics, vol. 31, SIAM, Philadelphia, 2000.

[18] C.-H. Lee, Q. H. Ansari, and J.-C. Yao, A perturbed algorithm for strongly nonlinear variational-like inclusions, Bull. Austral. Math. Soc. 62 (2000), no. 3, 417-426.

[19] G. Mastroeni, Gap functions for equilibrium problems, J. Global Optim. 27 (2003), no. 4, 411-426.

[20] S. R. Mohan and S. K. Neogy, On invex sets and preinvex functions, J. Math. Anal. Appl. 189 (1995), no. 3, 901-908.

[21] U. Mosco, Implicit variational problems and quasi variational inequalities, Nonlinear Operators and the Calculus of Variations (Summer School, University Libré de Bruxelles, Brussels, 1975), Lecture Notes in Math., vol. 543, Springer, Berlin, 1976, pp. 83-156.

[22] M. A. Noor, Invex equiibrium problems, to appear in J. Math. Anal. Appl.

[23] _ _ Preinvex functions and variational inequalities, J. Nat. Geom. 9 (1966), 63-76. 
[24]__ Variational-like inequalities, Optimization 30 (1994), no. 4, 323-330.

[25] __ Nonconvex functions and variational inequalities, J. Optim. Theory Appl. 87 (1995), no. 3, 615-630.

[26] _ Merit function for variational-like inequalities, Math. Inequal. Appl. 3 (2000), no. 1, 117-128.

[27] _ Multivalued general equilibrium problems, J. Math. Anal. Appl. 283 (2003), no. 1, 140-149.

[28] _ Theory of General Variational Inequalities, Lecture Notes, Etisalat College of Engineering, Sharjah, United Arab Emirates, 2003.

[29] _ Auxiliary principle technique for equilibrium problems, J. Optim. Theory Appl. 122 (2004), 371-386.

[30] _ Fundamental of mixed quasi variational inequalities, Int. J. Pure Appl. Math. 15 (2004), no. 2, 138-257.

[31]_ Generalized mixed quasivariational inequalities, Appl. Math. Comput. 156 (2004), $145-158$.

[32] _ Invex $\epsilon$-equilibrium problems with trifunction, Int. J. Pure Appl. Math. 13 (2004), no. 1, 123-136.

[33] _ Multivalued equilibrium problems with trifunction, Aust. J. Math. Anal. Appl. 1 (2004), no. 1, 1-11.

[34] _ On a class of nonconvex equilibrium problems, Appl. Math. Comput. 157 (2004), 653-666.

[35] M. A. Noor and K. I. Noor, On general mixed quasivariational inequalities, J. Optim. Theory Appl. 120 (2004), no. 3, 579-599.

[36] M. A. Noor and W. Oettli, On general nonlinear complementarity problems and quasiequilibria, Matematiche (Catania) 49 (1994), no. 2, 313-331.

[37] J. Parida and A. Sen, A variational-like inequality for multifunctions with applications, J. Math. Anal. Appl. 124 (1987), no. 1, 73-81.

[38] T. Weir and B. Mond, Pre-invex functions in multiple objective optimization, J. Math. Anal. Appl. 136 (1988), no. 1, 29-38.

[39] X. Q. Yang and G. Y. Chen, A class of nonconvex functions and pre-variational inequalities, J. Math. Anal. Appl. 169 (1992), no. 2, 359-373.

[40] D. L. Zhu and P. Marcotte, Co-coercivity and its role in the convergence of iterative schemes for solving variational inequalities, SIAM J. Optim. 6 (1996), no. 3, 714-726.

Muhammad Aslam Noor: Etisalat College of Engineering, P.O. Box 980, Sharjah, United Arab Emirates

E-mail address: noor@ece.ac.ae 


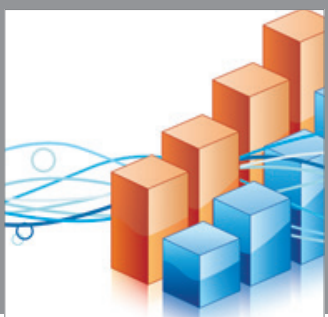

Advances in

Operations Research

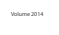

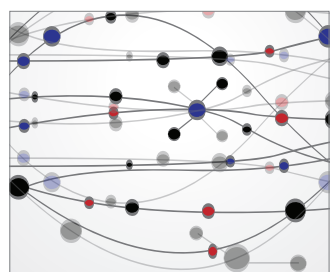

\section{The Scientific} World Journal
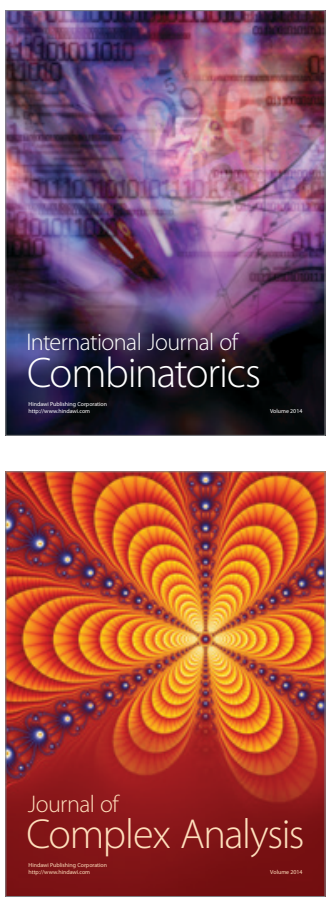

International Journal of

Mathematics and

Mathematical

Sciences
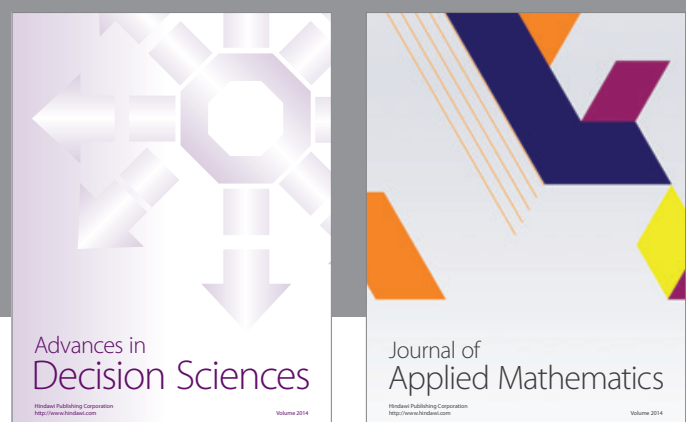

Journal of

Applied Mathematics
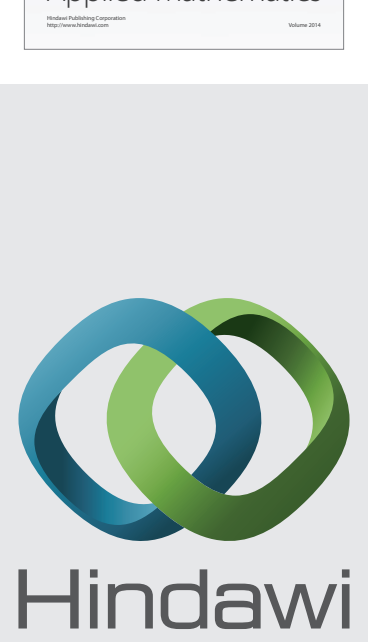

Submit your manuscripts at http://www.hindawi.com
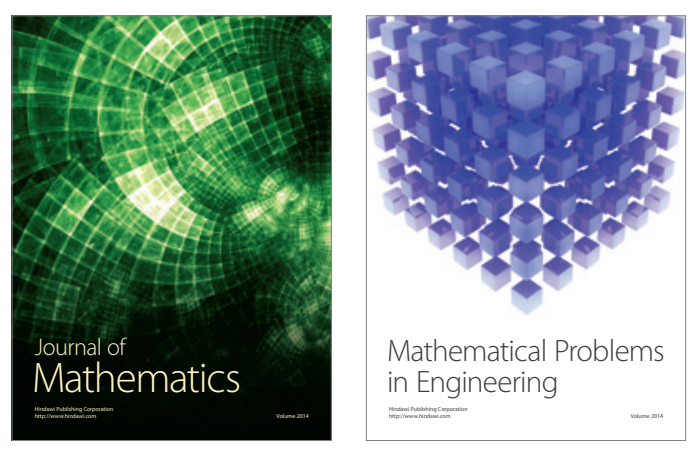

Mathematical Problems in Engineering
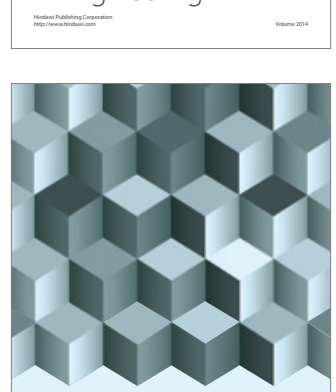

Journal of

Function Spaces
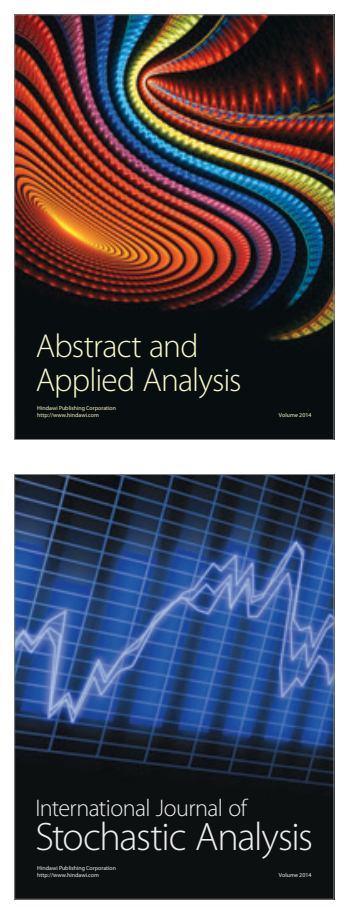

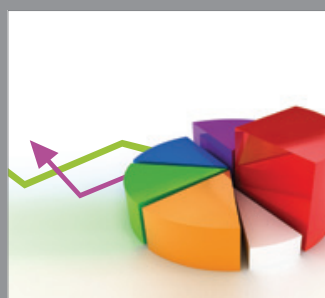

ournal of

Probability and Statistics

Promensencen
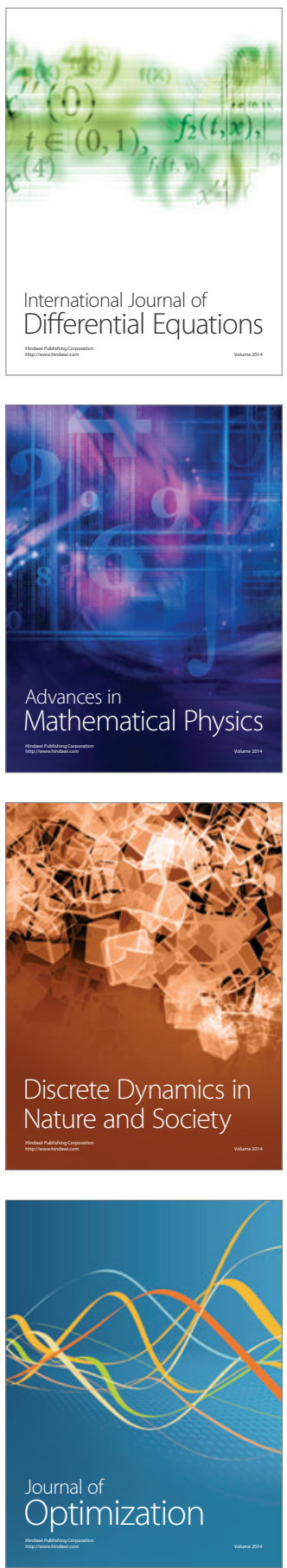